\title{
Visualization of cyclic nucleotide dynamics in neurons
}

\section{Kirill Gorshkov and Jin Zhang*}

Laboratory of Dr. Jin Zhang, Department of Pharmacology and Molecular Sciences, Johns Hopkins University School of Medicine, Baltimore, Maryland, USA

\section{Edited by:}

Pierre Vincent, Centre National de la

Recherche Scientifique, France

\section{Reviewed by:}

Bernd Kuhn, Okinawa Institute of

Science and Technology Graduate

University, Japan

Liliana Ribeiro Vivas Castro,

Université Pierre et Marie Curie,

France

Viacheslav Nikolaev, University of

Goettingen, Germany

\section{*Correspondence:}

Jin Zhang, Laboratory of Dr. Jin

Zhang, Department of

Pharmacology and Molecular

Sciences, Johns Hopkins University

School of Medicine, 725 North

Wolfe Street, Baltimore, Maryland,

21205, USA

e-mail: jzhang32@jhmi.edu
The second messengers cyclic adenosine monophosphate (cAMP) and cyclic guanosine monophosphate (cGMP) transduce many neuromodulatory signals from hormones and neurotransmitters into specific functional outputs. Their production, degradation and signaling are spatiotemporally regulated to achieve high specificity in signal transduction. The development of genetically encodable fluorescent biosensors has provided researchers with useful tools to study these versatile second messengers and their downstream effectors with unparalleled spatial and temporal resolution in cultured cells and living animals. In this review, we introduce the general design of these fluorescent biosensors and describe several of them in more detail. Then we discuss a few examples of using cyclic nucleotide fluorescent biosensors to study regulation of neuronal function and finish with a discussion of advances in the field. Although there has been significant progress made in understanding how the specific signaling of cyclic nucleotide second messengers is achieved, the mechanistic details in complex cell types like neurons are only just beginning to surface. Current and future fluorescent protein reporters will be essential to elucidate the role of cyclic nucleotide signaling dynamics in the functions of individual neurons and their networks.

Keywords: biosensor, neuron, FRET, fluorescence, signaling, cyclic nucleotide, cAMP, cGMP

\section{INTRODUCTION}

The cyclic nucleotides cyclic adenosine monophosphate (cAMP) and cyclic guanosine monophosphate (cGMP) are ubiquitous second messengers present in most cell types. Within the brain, cyclic nucleotides transduce neuromodulatory signals into functional outputs for individual neurons leading to changes in neural networks themselves or their function. The importance of cyclic nucleotide signaling pathways is well appreciated in the field of clinical neuroscience and psychiatry, with many drugs targeting the G-protein coupled receptors to modulate neuronal activity (Lim, 2007; Taly, 2013). The effects of cAMP and cGMP signaling range from regulating neuronal differentiation and growth to axonal guidance and modulation of neuronal excitability. To accomplish this, cyclic nucleotides are coupled to many downstream effectors (Antoni, 2000). cAMP, the prototypical cyclic nucleotide, transduces G-protein signals to activate protein kinase A (PKA) and exchange protein activated by cAMP (Epac). cGMP, on the other hand, relays signals from nitric oxide to activate protein kinase G (PKG). Phosphodiesterase (PDE), the enzyme that degrades cGMP, can be a cGMP effector with its activity modulated by cGMP binding to regulatory domains forming feedback loops (Conti and Richter, 2014). Both cyclic nucleotides also activate cyclic nucleotide gated ion channels (Rich et al., 2014).

The idea of compartmentalized signaling is an essential component to the cyclic nucleotide signaling model. To elicit their

Abbreviations: cyclic nucleotide binding domain, CNBD; Dopamine 1 receptor, D1R; light-oxygen-voltage, LOV; sensors of blue light using FAD, BLUF. diverse functional effects in a highly specific manner, cAMP and cGMP signaling is thought to be spatially compartmentalized (Zaccolo and Stangherlin, 2014). The levels of cyclic nucleotides and the activities of downstream effectors are not uniform throughout the cell, but instead form specific nanodomains or microdomains inside the cell. The spatial compartmentation is achieved, at least partially, by strict regulation of cyclic nucleotide production and degradation. cAMP is produced by adenylyl cyclase (AC) and cGMP is produced by guanylyl cyclase (GC). These enzymes are located within the plasma membrane as transmembrane proteins, or within the cytoplasm or organelles as soluble forms of the enzyme. The degradation of cAMP and cGMP is carried out by PDEs which have specificity for cAMP, cGMP, or both. PDEs have been shown to function as cAMP and cGMP sinks to help maintain these microdomains (Terrin et al., 2006; Biswas et al., 2008). The tight spatiotemporal regulation of cAMP signaling is achieved with the help of the A-kinase anchoring protein (Esseltine and Scott, 2013), which assembles signaling complexes consisting of members of the cAMP/PKA signaling pathway like ACs, PDEs, PKA and its substrate, and other effectors. These signalosomes, which can be found throughout various compartments including the plasma membrane, the cytosol and the nucleus, have been shown to play important roles in achieving functional specificity of the cAMP/PKA pathway. In addition to biochemical regulation, the structural properties of cells can also affect the signaling dynamics of second messengers (Castro et al., 2010; Neves, 2012).

Relatively recent advances in fluorescent biosensor technology allow researchers to track the dynamics of cyclic nucleotides and 
their effectors in living neurons and brain tissue. Here we will describe several genetically encoded fluorescent biosensors for monitoring cyclic nucleotide dynamics. Then we will focus on a few studies that demonstrate their implementation in living neurons for the purpose of understanding how cyclic nucleotides impact neuronal function. We will end with a discussion of novel tools and modeling efforts that help us understand neuronal cyclic nucleotide dynamics.

\section{GENETICALLY ENCODED FLUORESCENT BIOSENSORS}

The genetically encoded fluorescent biosensors described in the following sections allow for the continuous monitoring of free cyclic nucleotides with high spatiotemporal resolution. These biosensors are engineered based on a general design: a sensing unit to detect the change in free cyclic nucleotide concentration and a reporting unit to convert the biochemical change into a fluorescent readout. The sensing unit for cyclic nucleotide biosensors is one or more cyclic nucleotide binding domains (CNBD). The reporting unit can be made up of two fluorescent proteins flanking the sensing unit as is the typical arrangement for fluorescence resonance energy transfer (FRET)-based biosensors. In FRET, energy is transferred non-radiatively from an excited donor molecule to an acceptor molecule. For a fixed FRET donor-acceptor pair, the efficiency of FRET is dependent on the distance and orientation of the two fluorophores. In a FRET-based biosensor, cyclic nucleotide binding induces conformational changes of the CNBD sensing unit, which acts as a molecular switch to change the physical separation or orientation between the fluorescent protein pair resulting in a change in FRET (Figure 1A). In intensity-based biosensors, on the other hand, the reporting unit can be a single fluorescent protein. In this case, conformational changes in the sensing unit are translated into changes in fluorescence intensity (Figure 1B). Different sensors vary in their CNBD sensing units and fluorescent protein reporting units. For a thorough overview of fluorescence and fluorescent proteins used in many different types of biosensors, please refer to the reviews by Sample et al. (2009); Newman et al. (2011). The following subsections describe the various designs of many cAMP and cGMP reporters. Please refer to Table $\mathbf{1}$ for a detailed list of the biosensors described here.

\section{FLUORESCENT INDICATORS OF CAMP}

\section{cAMP reporter based on dissociation of PKA holoenzyme}

For the purposes of tracking CAMP, researchers have been developing molecular biosensors for the past several decades. The first of such biosensors called FlCRhR (Fluorescein-labeled PKA Catalytic subunit and Rhodamine-labeled Regulatory subunit) was based on chemically labeled regulatory and catalytic subunits of the PKA holoenzyme (Adams et al., 1991). Upon cAMP binding the catalytic and regulatory subunits dissociate producing a change in FRET. This labeled holoenzyme was microinjected into living cells and imaged under widefield microscopy. A genetically encodable version was generated allowing for expression in a wide array of cell types, thereby expanding the scope of the application (Zaccolo et al., 2000). The fusion protein contained an 11 amino acid linker between the PKA regulatory subunit and the fluorescent proteins (RII-EBFP; C-GFP ${ }^{S 65 T}$ ). A newer version was generated using CFP fused to the RII regulatory domain and YFP fused to the catalytic domain (Zaccolo and Pozzan, 2002; Mongillo et al., 2004). By using molecular dynamics simulations, this design was further improved with the introduction of a longer, more rigid 20 amino acid linker (Lissandron et al., 2005). Live-cell fluorescent lifetime and acceptor sensitized measurements of this sensor showed a doubling of the dynamic range.

\section{Single-chain FRET-based cAMP indicators}

Multimeric biosensors like RII-CFP/C-YFP require equal expression of both subunits to form the PKA tetramer. In addition, there can be interactions with endogenous PKA subunits which do not make contributions to the FRET response. On the other hand, single chain biosensors offer ease of use and targeting to subcellular locations, increasing the spatial resolution offered by FRETbased biosensors. In 2004, three groups independently developed single-chain cAMP FRET sensors using the CNBD from Epac, a Rap1 guanine nucleotide exchange factor, or PKA. The reported affinities for cAMP range from $\sim 0.3$ to $50 \mu \mathrm{M}$. However, we note that experimental variations such as biosensor concentrations when determining $\mathrm{EC}_{50}$ s could largely affect the resulting values. We include the experimentally determined $\mathrm{EC}_{50} \mathrm{~s}$ in Table 1 but these values may not accurately reflect the differences in biosensor affinities.

In Epac1-camps, the cAMP binding domain of the Epac1 protein (Epac1 ${ }^{157-316}$ ) was flanked by an N-terminal CFP and a C-terminal YFP (Nikolaev et al., 2004). Epac2-camps used the cAMP binding domain B from Epac2 (Epac2B ${ }^{284-443}$ ) instead. Both biosensors generated a decrease in the yellow to cyan emission ratio upon binding CAMP, indicating a decrease in FRET. In an attempt to generate a more sensitive cAMP probe called Epac2-camps300, Norris et al. introduced a K405E mutation into Epac2-camps that decreased the $\mathrm{EC}_{50}$ from $\sim 0.9 \mu \mathrm{M}$ to $\sim 0.3 \mu \mathrm{M}$ (Norris et al., 2009). Nikolaev et al. also generated a single chain PKA-camps using a portion the PKA regulatory $\beta$ II subunit $(\mathrm{RII} \beta)$ sandwiched between ECFP and EYFP (Nikolaev et al., 2004). PKA-camps incorporated the cAMP binding domain B from amino acids 264-403 of RII $\beta$ as the sensing unit. Upon binding cAMP, RII $\beta$ undergoes a conformational change conducive to a change in FRET. These single CNBD containing biosensors are the smallest cAMP probes currently available.

A series of Epac based reporters called ICUE (Indicator of cAMP Using Epac) have been developed in parallel. First in the series, ICUE1 contained full-length Epac1 sandwiched between ECFP and the YFP variant Citrine (DiPilato et al., 2004). Like Epac1-camps and Epac2-camps, ICUE also responds to cAMP with a decrease in the yellow to cyan emission ratio. ICUE2, an improved version of ICUE1, has an $\mathrm{EC}_{50}$ of $\sim 12.5 \mu \mathrm{M}$ and contains a $\mathrm{N}$-terminally truncated Epac1 protein (Epac1 ${ }^{149-881}$ ) (Violin et al., 2008). This biosensor showed improvement in localization over ICUE1 due to removal of a membrane and mitochondria targeting sequence located at the N-terminus. More recently, we developed ICUE3 with an increased dynamic 


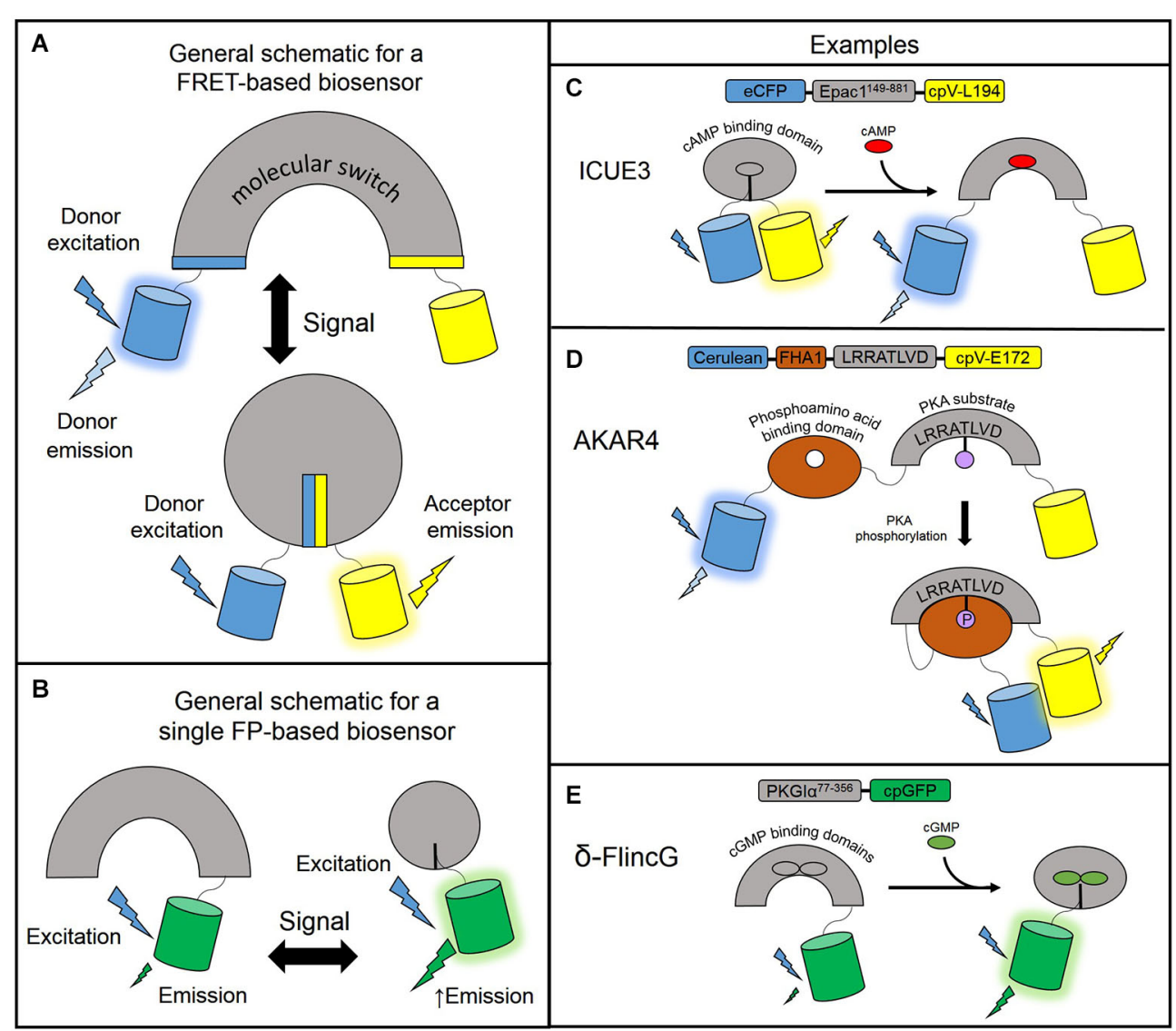

FIGURE 1 | Designs of genetically encodable biosensors. (A) General design of a FRET-based biosensor consisting of a sensing unit which acts as a molecular switch to change the distance or orientation of the fluorescent protein reporting unit. In a low FRET situation, the donor fluorescent protein is excited and emits light at its own wavelength. In the high FRET situation, donor excitation allows for resonance energy transfer to the acceptor fluorescent protein which emits light at its own wavelength. (B) General design of a single fluorescent protein-based biosensor. The sensing unit transduces a signal via a conformational change to the linked fluorescent protein reporting unit which undergoes its own conformational change and modulates fluorescence intensity. A circularly permuted GFP is often used to enhance the change in fluorescence. (C) ICUE3 consists of an Epac1 $1^{149-881}$ sensing unit flanked by an ECFP donor and a cpV-L194 acceptor reporting unit. Upon binding CAMP, the sensor switches from a high FRET to a low FRET

conformation. (D) AKAR4 contains a sensing unit consisting of a FHA1 phosphoamino acid binding domain and a substrate peptide. The reporting unit is comprised of a Cerulean donor and cpV-E172 acceptor reporting unit. When PKA activity is high, the substrate peptide is phosphorylated and binds the FHA1 domain to induce FRET. (E) $\delta$-FlincG utilizes a PKG1 $\alpha^{77-356}$ sensing unit linked to a single cpGFP reporting unit. Upon binding $C G M P$, the fluorescence intensity of cpGFP increases. range by changing the FRET acceptor Citrine to a circularly permuted Venus at lysine 194 (cpV-L194; DiPilato and Zhang, 2009; Figure 1C). The large dynamic range of ICUE3 ( 100\% emission ratio change) makes it suitable for subcellular targeting for detecting local cAMP changes (e.g., plasma membrane and nucleus (Sample et al., 2012), sarcoplasmic reticulum (Liu et al., 2012), primary cilia (Marley et al., 2013)) as addition of subcellular localization tags sometimes leads to decreased response amplitudes.

The Jalink lab developed a similar biosensor CFP-Epac( $\delta$ DEPCD)-YFP using Epac1 ${ }^{149-881}$ flanked by an amino-terminal CFP and carboxy-terminal YFP (Ponsioen et al., 2004). Mutations T781A and F782A were introduced to generate a catalytically dead (CD) Epac1. The lab has recently developed newer versions, some of which are useful for fluorescence lifetime imaging FRET (FLIM-FRET) measurements (Klarenbeek et al., 2011; Polito et al., 2013). Named Epac-S ${ }^{H 150}$, the newest version in this series incorporated the Q270E mutation (Dao et al., 2006) to increase the affinity for cAMP (EC $\left.{ }_{50} 4 \mu \mathrm{M}\right)$ and used mTurquoise 2 as the FRET donor and a single circularly-permuted Citrine as the FRET acceptor (Polito et al., 2013).

In some cases, the concentration of cAMP in a cell can saturate a highly sensitive reporter. To accommodate large fluctuations in signaling, Nikolaev et al. used the hyperpolarization-sensitive cyclic nucleotide gated channel 2 (HCN2) to generate HCN2-camps and image large changes in cAMP concentration in cardiomyocytes (Nikolaev et al., 2006a). In the context of probes like PKA-camps, Epaccamps, and CFP-RII/C-YFP, this probe exhibited a lower affinity for $\mathrm{cAMP}\left(\mathrm{EC}_{50} \sim 6 \mu \mathrm{M}\right)$ and provides another tool to monitor cAMP in cells with larger basal or fluctuating concentrations. 


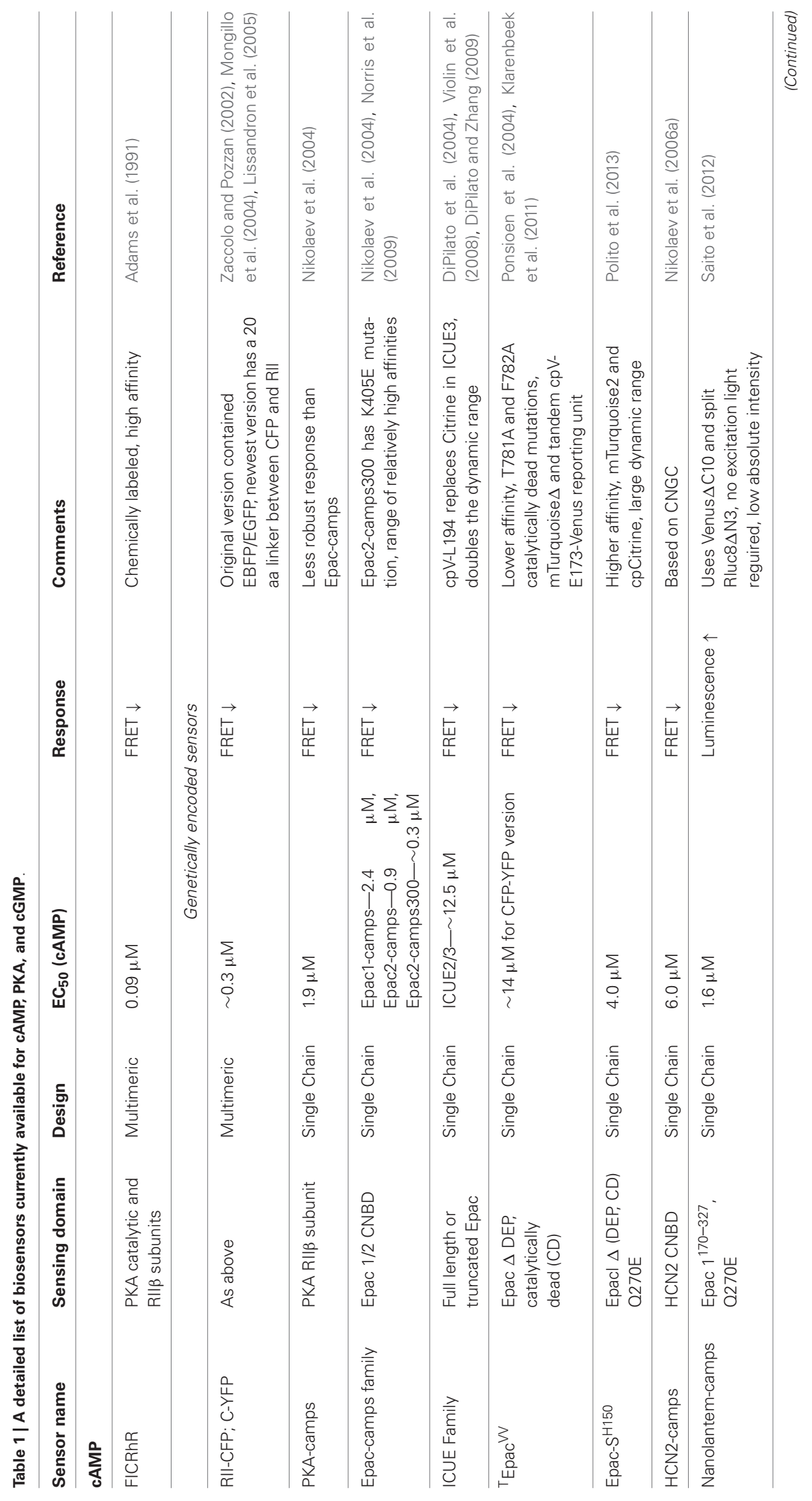




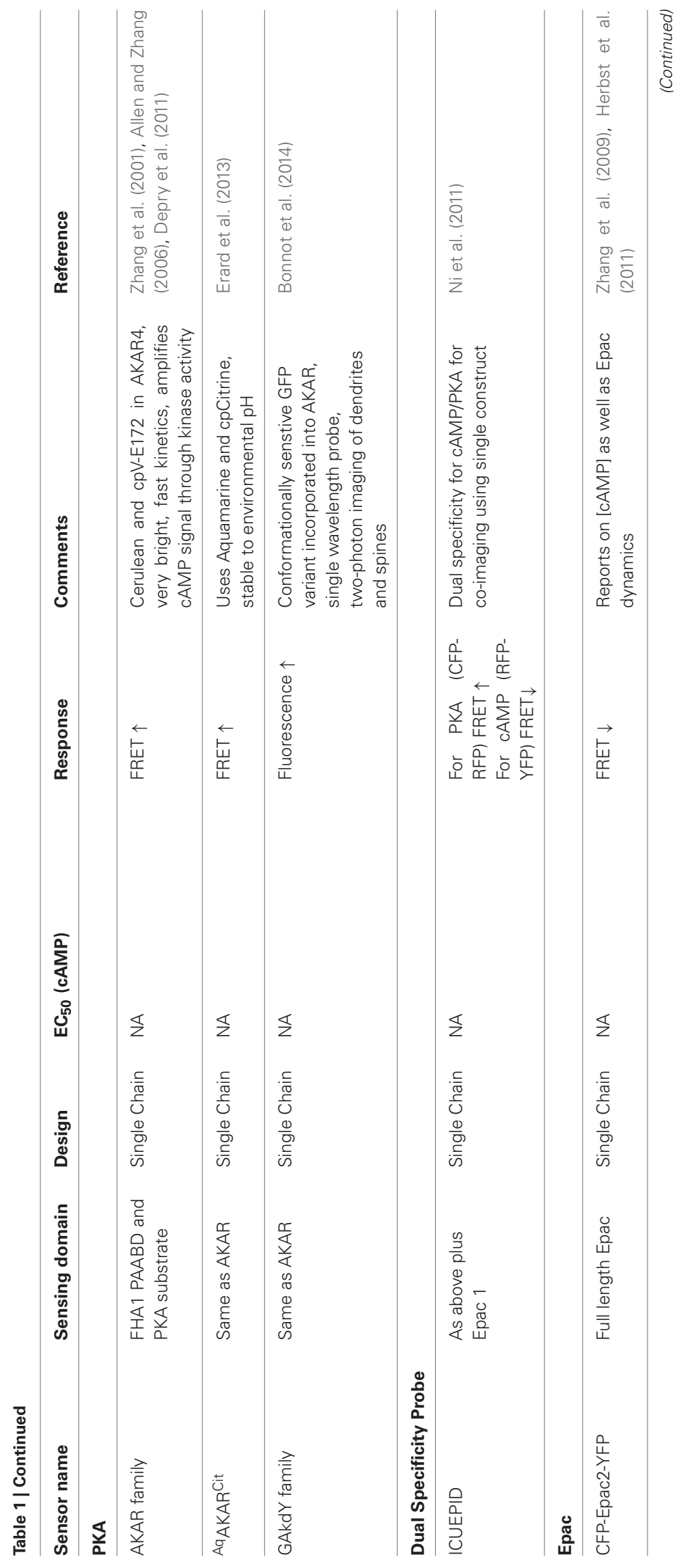




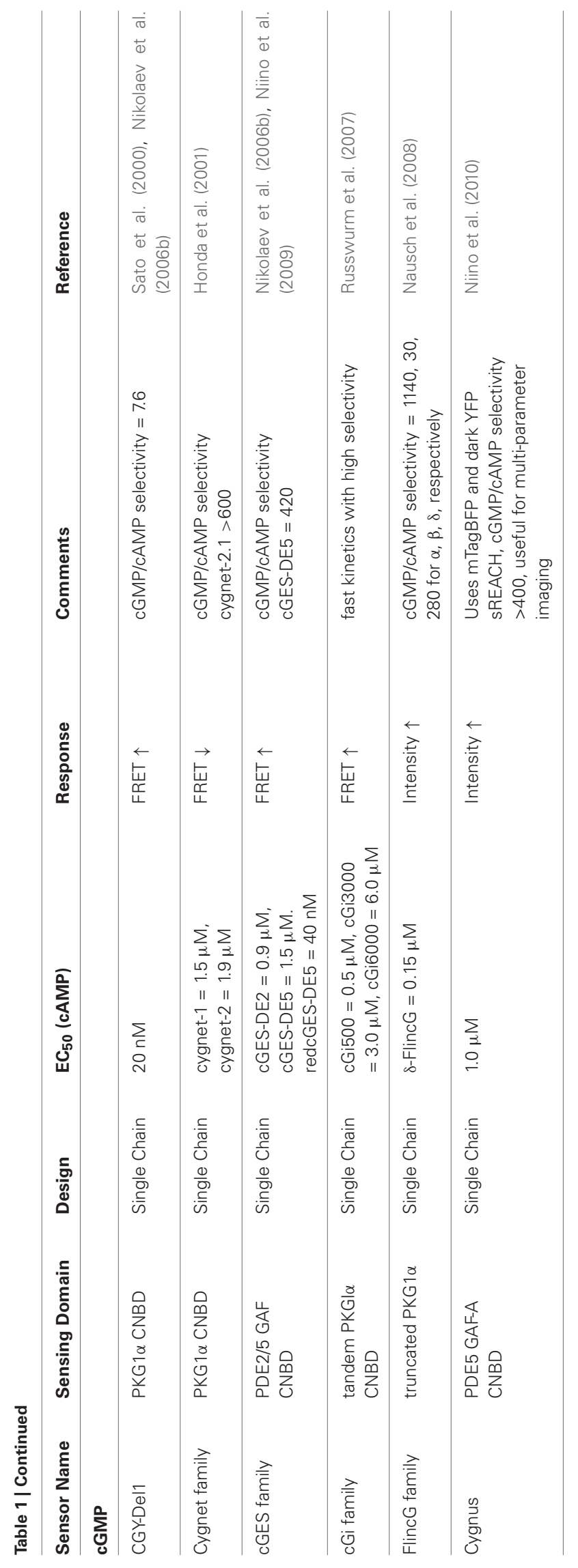




\section{Indicators of downstream cAMP effectors}

Neuromodulatory signals can be transduced by cAMP and cGMP through their downstream target kinases PKA and PKG, respectively (Giese and Mizuno, 2013). Although there are no biosensors yet available for PKG activity, the A-kinase activity reporter (AKAR) can report on the kinase activity of PKA (Zhang et al., 2001). AKAR uses a molecular switch consisting of a phosphoamino acid binding domain linked to a PKA-specific substrate sequence, flanked by CFP and YFP. PKA phosphorylation of its substrate induces binding of the phosphorylated substrate to the phosphoamino acid binding domain, leading to an increase in FRET. AKAR1 displayed an irreversible FRET response which prevented continuous monitoring of PKA dynamics. This was presumably due to the high affinity of the 14-3-3 binding domain for the substrate as tight binding may prevent phosphatases from dephosphorylating the substrate and reversing the FRET response. This was overcome by the generation of AKAR2 which utilized the lower binding affinity forkhead-associated domain 1 (FHA1) and exhibited a reversible FRET response (Zhang et al., 2005). The kinetics of AKAR2 were improved in AKAR2.2 by replacing the dimeric forms of ECFP and Citrine with versions that resist dimerization. The dynamic range was doubled in AKAR3 by replacing the YFP acceptor in AKAR2 with cpVE172 (Allen and Zhang, 2006). The dynamic range of AKAR was further enhanced with latest version, AKAR4, by replacing ECFP with Cerulean (Depry et al., 2011; Figure 1D). Due to the amplification of the cAMP signal by PKA phosphorylation activity, PKA activity reporters may be able to detect signals not picked up by cAMP binding probes. Recently, a modified AKAR probe named ${ }^{A q} \mathrm{AKAR}^{C i t}$, was generated by replacing Cerulean with Aquamarine, a newly engineered CFP variant, which has mutations T65S and H148G (Erard et al., 2013). These modifications to ECFP increased its photophysical properties and reduced its environmental sensitivity to low $\mathrm{pH}$.

The modular design of FRET-based biosensors allows researchers to couple the same sensing unit to different reporting units. For example, red fluorescent protein (RFP) can act as an acceptor for both CFP and YFP. ICUE2 was redesigned as a YFPRFP FRET sensor (YR-ICUE) and co-imaged with a CFP-RFPAKAR (CR-AKAR) to analyze the temporal relationship between cAMP and PKA signaling following receptor activation in HEK293 cells (Aye-Han et al., 2012). Utilizing the CFP-RFP and YFPRFP based biosensors for co-imaging is a simple technique to monitor two biochemical events in parallel and only requires the addition of an RFP emission filter to the imaging setup. With the shared RFP receptor, these two biosensors were further combined to generate the single-chain dual-specificity probe ICUEPID which can sense both CAMP and PKA simultaneously (Ni et al., 2011). It utilizes CFP and YFP donors and a single RFP acceptor. The PKA activity sensing unit is flanked by CFP and RFP while the cAMP sensing unit is flanked by RFP and YFP. This sensor proved useful in Min6 pancreatic $\beta$-cells to detect synchronized oscillations of cAMP and PKA within the same subcellular location.

A single-wavelength PKA activity sensor was designed by utilizing a conformation sensitive GFP variant and combining it with the sensing unit of AKAR2 to generate GAkdY (Bonnot et al., 2014). PKA phosphorylation of the substrate changes the conformation of the probe and modulates GFP fluorescence intensity and lifetime. Single color activity sensors such as this probe provide a way to image multiple kinase activities with single excitation wavelengths for each sensor. Three of these sensors were incorporated into Sindbis viral vectors and expressed in brain slices to visualize PKA dynamics in pyramidal cell bodies, thin dendrites, and dendritic spines using two-photon microscopy.

Indicators of enzyme activation typically have sensing domains comprised of the full length endogenous protein. Epac1 and Epac2 share similar activation mechanisms whereby cAMP binding relieves the steric block of the regulatory domain on the Rap1 binding catalytic site by inducing a conformational change in the regulatory domain hinge helix (Selvaratnam et al., 2012). In this context, ICUE1 is also an Epacl activation probe. A FRET reporter for Epac2 activation was generated by sandwiching full length Epac2 between ECFP and EYFP (Zhang et al., 2009). A brighter Epac2 activation reporter is also available containing Cerulean and Venus, variants of CFP and YFP, respectively (Herbst et al., 2011).

\section{FLUORESCENT INDICTORS OF cGMP}

Cyclic guanosine monophosphate probes, like cAMP probes, report the presence of cGMP by using a CNBD derived from PKG or cGMP-specific PDEs. Because the concentration of cGMP in neurons is lower than cAMP, the probes must be highly sensitive and specific in order to provide a high signal to noise ratio and large dynamic range.

\section{Single-chain FRET-based indicators of cGMP}

The cygnet series of cGMP reporters was developed by the Dostmann lab and utilized both CNBDs from PKG1 $\alpha$ as a sensing unit that responds to cGMP (Honda et al., 2001). Therefore, each molecule of cygnet binds two molecules of cGMP. In cygnet-1 (cyclic GMP indicator using energy transfer), the first 77 amino acids of PKG1 $\alpha$ (PKG1 $\left.\alpha^{\Delta 1-77}\right)$ were truncated. PKG $1 \alpha^{\Delta 1-77}$ was flanked by a reporting unit comprised of ECFP and EYFP at the $\mathrm{N}$ - and C- termini, respectively. Cygnet-2, the catalytically inactive version of cygnet-1, was generated by introducing mutation T516A to $\mathrm{PKG} 1 \alpha^{\Delta 1-77}$. The $\mathrm{pH}$-insensitive EYFP variant Citrine was used to replace YFP in cygnet-2 to generate cygnet-2.1. cGMP binding to the cygnet reporters induces a decrease in FRET. Cygnet- 1 and cygnet-2 have an affinity of $1.5 \mu \mathrm{M}$ and $1.9 \mu \mathrm{M}$ for cGMP, respectively, although endogenous PKG1 $\alpha$ has a cGMP affinity of $\sim 100 \mathrm{nM}$. It is conceivable that fusion of the fluorescent proteins or the $\Delta 1-77 \mathrm{~N}$-terminal truncation and catalytic site mutations affected their cGMP binding affinity. Around the same time, Sato et al. generated a similar PKG1 $\alpha$ based probe called CGY-Del1 which had an N-terminal truncation of the first 47 amino acids (Sato et al., 2000). cGMP binding induces an increase in FRET in this probe.

Nikolaev et al. developed smaller probes utilizing a single CNBD from PKG1 $\alpha^{231-350}$, PDE2 $2^{392-525}$ and PDE5 $5^{154-308}$ (Nikolaev et al., 2006b). Their efforts culminated in the generation of three sensors, cGES-GKIB (for cGMP energy transfer sensor derived from GKI-B (PKGI) site), cGES-DE2 (derived from PDE2A), and cGES-DE5 (derived from PDE5A). All three 
FRET sensors had reporting units consisting of EYFP and ECFP at the $\mathrm{N}$ - and $\mathrm{C}$ - termini, respectively. Interestingly, cGES-GKIB exhibited a cGMP-dependent decrease in FRET whereas cGESDE2/5 exhibited a cGMP-dependent increase in FRET. These three probes all exhibited strong FRET responses, but cGES-DE5 containing the GAF-A domain of PDE5A had a $\sim 400-600$ fold greater selectivity for cGMP:cAMP making it the preferred cGMP sensor for live-cell applications. More recently, a red version of cGES-DE5 was generated for co-imaging experiments with CFPYFP sensors by using the GFP variant T-SapphireC $\Delta 11$ and RFP dimer2 (Niino et al., 2009). Surprisingly, switching the CFP-YFP to GFP-RFP variants increased the affinity of cGES-DE5. The large enhancement in the probe affinity $\left(\mathrm{EC}_{50} \sim 40 \mathrm{nM}\right)$ makes it potentially suitable for detecting low cGMP concentrations but may also be confounded by variations in experimental conditions when determining the $\mathrm{EC}_{50}$. The probe was used to develop a method for simultaneous measurements of signaling activities by using a single excitation light that excites both T-sapphire and CFP, four channel detection and linear unmixing.

Russwurm and colleagues generated a series of FRET based cGMP sensors in order to achieve faster kinetics and provide an array of probes with a range of affinities (Russwurm et al., 2007). These sensors used the tandem CNBD domains from PKGI $\alpha$ as their sensing unit. Beginning with the indicator construct CFP-PKGI $\alpha^{79-336}$-YFP, they elongated the N- and C- termini of PKGI $\alpha^{79-336}$ and screened for constructs based on cGMP affinity and the FRET response amplitude. Using this approach, they generated a series of cGMP biosensors cGi-500 $\left(\mathrm{EC}_{50}=500 \mathrm{nM}\right)$, $\mathrm{cGi}-3000\left(\mathrm{EC}_{50}=3.0 \mu \mathrm{M}\right)$, and cGi-6000 $\left(\mathrm{EC}_{50}=6.0 \mu \mathrm{M}\right)$.

\section{Single-FP based cGMP sensors}

Nausch et al. generated a line of biosensors called fluorescent indicators of cGMP (FlincG; Nausch et al., 2008). The reporting unit of FlincG contains a single circularly-permuted EGFP (cpEGFP) molecule. In this series, complete or truncated cGMP binding regulatory domains from PKG1 were used to construct the sensing unit. First in line, $\alpha$-FlincG used the complete regulatory domain of PKG1 $\alpha^{1-356}$. Second, $\beta$-FlincG contained the complete regulatory domain from PKG1 $\beta$, which has an activation constant of $1.0-1.8 \mu \mathrm{M}$ compared to $75 \mathrm{nM}$ for PKG1 $\alpha$, fused to the $\mathrm{N}$-terminus of cpEGFP. PKGI $\beta$ has a completely different $\mathrm{N}$ terminus than PKG1 $\alpha$, which highlights the importance of the $\mathrm{N}$-terminus for cGMP binding affinity. Lastly, the researchers removed the entire $\mathrm{N}$-terminal domain, the first 77 amino acids of PKG1 $\alpha$, to generate $\delta$-FlincG (Figure 1E). This decreases the $K_{D}$ of PKG1 $\alpha^{\Delta 1-77}$ to $\sim 170 \mathrm{nM}$. Because $\delta$-FlincG had a superior dynamic range and retained nanomolar affinity for cGMP in living cells, it was chosen as the preferred single-GFP linked cGMP biosensor for further characterization and application. Singlecolor sensors with adequate spectral separation allow for multiparameter imaging of interacting molecules in complex signal transduction networks. In addition to the green cGMP sensor described above, a blue single-color cGMP sensor named Cygnus was developed by using a blue fluorescent protein (BFP) and a dark fluorescent protein acceptor (Niino et al., 2010). This biosensor was generated by sandwiching the GAF-A domain of PDE5 between mTagBFP and the quenching acceptor YFP
sREACH. Cygnus was used to demonstrate cGMP imaging in rat hippocampal neurons and triple parameter imaging of $\mathrm{Ca}^{2+}$, cAMP, and cGMP in HEK-293T cells.

\section{APPLICATION OF CYCLIC NUCLEOTIDE BIOSENSORS TO STUDY NEURONAL SYSTEMS}

The following section highlights a few studies that utilize cyclic nucleotide biosensors in investigating neuronal polarization, axon guidance and growth, signaling, and plasticity.

\section{POLARIZATION}

Cyclic adenosine monophosphate and PKA are one of the few bona fide axon determinants that play a critical role in axon polarization (Cheng and Poo, 2012). In a recent study, Shelly et al. investigated the contributions of cAMP and cGMP to the process of axon and dendrite formation of early stage hippocampal neurons in isolated cultures. Given that cAMP and cGMP exerted opposing actions in other cell systems, it was possible that they played some role in the differentiation of neuronal processes to form distinct compartments. It was discovered that neurites exposed to cAMP have a high probability of differentiating into axons and those exposed to cGMP become dendrites (Shelly et al., 2010). But how are these processes coordinated in a single cell to ensure that only one neurite becomes the axon? Using the fluorescent biosensors ICUE and cGES-DE5 the researchers examined the effects of locally stimulating a single neurite with a glass bead soaked in cAMP agonist or cGMP analog. Local elevation of cAMP in one of the neurites resulted in a decrease of CAMP and increase of cGMP at the other neurites. Locally elevating cGMP only decreased cAMP at the stimulated neurite and did not exhibit long range inhibition of cGMP. They concluded that local and long range reciprocal regulation of cAMP and cGMP ensures the development of a single axon and multiple dendrites, although the exact mechanism of long range inhibition remains to be elucidated.

The question still stands as to which endogenous factors act through cAMP and cGMP to induce a single neurite to become an axon. In a follow-up study, Shelly et al. examined the effects of Semaphorin3A (Sema3A), a secreted molecule that guides axon/dendrites growth and neuronal migration (Shelly et al., 2011). Here, the researchers utilized the biosensors cGES-DE5, ICUE, and AKAR to monitor the effects of Sema3a and BDNF on cAMP and cGMP. Bath application of Sema3A led to a decrease in the levels of cAMP and PKA activity and an increase in cGMP. Bath application of BDNF led to the opposite changes in cAMP, PKA, and cGMP. Furthermore, blocking soluble guanylyl cyclase (sGC) and PKG with small molecule inhibitors prevented the increase in cGMP by Sema3A, indicating that Sema3A exerts its effects via $P K G$ regulation of sGC. The same compounds prevented the Sema3A induced decrease in cAMP. These results suggest that Sema3A and BDNF exert opposing actions on axondendrite differentiation mediated through reciprocal regulation of cyclic nucleotides, consistent with their previously reported findings (Shelly et al., 2010). This study revealed Sema3a's role as a polarizing factor which favors the differentiation of neurites to dendrites while suppressing axon formation in cultured hippocampal neurons. 


\section{GROWTH}

Cyclic adenosine monophosphate probes can be used to dissect the specific contributions of cAMP modulating GPCRs to physiological changes like axon growth. ICUE3 was recently used to investigate the impact of ionotropic and metabotropic purinergic receptor signaling on axon elongation (del Puerto et al., 2012). Metabotropic P2Y receptors are activated by ADP, whereas the ionotropic P2X receptors are activated by ATP. By modulating purinergic signaling pathways in cultured hippocampal neurons, the authors found that $\mathrm{P} 2 \mathrm{Y} 1$ enhances axonal elongation while P2Y13 and P2X7 halt axonal elongation. Addition of ADP or a $\mathrm{P} 2 \mathrm{X} 7$ antagonist increased cAMP levels in the distal region of the axon as reported by ICUE3. Concurrent application of an AC5 inhibitor prevented the CAMP increase. Therefore, the purinergic receptors regulate $\mathrm{CAMP}$ levels through AC5 and regulate axonal elongation triggered by neurotrophic factors.

\section{GUIDANCE}

Cyclic adenosine monophosphate is a second messenger that has long been appreciated in guiding axon elongation as the first step in neural circuit formation. The high spatial and temporal resolution of FRET based biosensors allows researchers to study transient cAMP signals in restricted areas of the neuron such as the growth cone, a structure at the tip of the growing axon that regulates axonal growth and pathfinding. Epac2-camps was used extensively in a study that investigated the spatial and temporal dynamic interactions between cAMP and calcium within the axonal growth cone and its filopodia (Nicol et al., 2011). By fusing the sensor to an N-terminal plasma membrane localization signal (pm-Epac2-camps), the researchers were able to reduce differences in fluorescence intensity between the filopodia and growth cone center allowing for direct comparison of FRET signals. First, the authors showed that bath application of the axon guidance molecule Netrin-1 induces cAMP and calcium transients with similar kinetics. Next, by modulating AC, they were able to show that Netrin-1 induced calcium transients in growth cone filopodia are driven by transient elevation of cAMP. The growth cone center, on the other hand, was shown to have a delayed and prolonged calcium driven cAMP increase as compared to the filopodia. Therefore, the cAMP in the growth cone center is downstream of calcium, opposite that of the filopodia.

\section{SIGNALING AND EXCITABILITY}

Genetically encoded biosensors have provided direct evidence for signaling compartmentalization in neurons. For example, the AKAR biosensor targeted to nuclear and cytosolic compartments revealed that PKA signaling within the nucleus of thalamic intralaminar neurons is delayed four-fold as compared to the cytosol (Gervasi et al., 2007). This data is consistent with the view that PKA signals propagate slowly to the nucleus via diffusion of the PKA catalytic subunit (Harootunian et al., 1993). Additionally, the thin dendrites of mouse cortical neurons exhibit larger cAMP and PKA responses than the bulk cytosol (Castro et al., 2010). This data corresponds well to modeling predictions of stronger CAMP and PKA signals in regions which have higher surface area to volume ratios (Neves, 2012). Castro et al. further compared CAMP/PKA responses in mouse brain slices triggered by dopamine D1 receptors in the cortex and the striatum (Castro et al., 2013). Biosensor imaging in pyramidal cortical neurons and striatal medium spiny neurons (MSNs) showed that CAMP/PKA response was stronger, faster, and longer lasting in the striatum than the cortex. They attributed this to more active PDE4 in the cortex, stronger AC activity in the striatum, and the phosphatase inhibitor DARPP-32 in the striatum which prolong the effects of PKA substrate phosphorylation. Thus, the PKA signaling cascade exhibits differential integration of upstream modulators within different brain areas and cell types.

Signaling crosstalks can also be dissected using fluorescent biosensors. Polito et al. used Cygnet2 and Epac- $\mathrm{S}^{H 150}$ to monitor cGMP and cAMP, respectively in medium spiny neurons of the striatum (Polito et al., 2013). They studied the NO response in striatonigral and striatopallidal MSNs and found that it was partially controlled by PDE2. D1 and D2 MSNs were found to have different transient cAMP responses to brief Fsk stimulation. PDE2 activation by cGMP prevented these cAMP responses and was magnified at the level of PKA activity as visualized by the AKAR3 biosensor. Therefore, PDE2 is a critical effector of NO and modulates the post-synaptic response of MSNs to dopaminergic transmission.

The application of fluorescent biosensors has also provided new insights into signaling mechanisms. It is known that dopamine D1 receptors undergo rapid endocytosis following agonist induced activation. In another recent study, researchers from the von Zastrow lab set out to investigate the functional significance of dopamine receptor endocytosis using the Epac1camps biosensor in cultured striatal neurons (Kotowski et al., 2011). In this study, internalization of a pH-sensitive fluorescently tagged D1R was tightly coupled $(<1 \mathrm{~min}$.) to an increase in cAMP upon stimulation with the D1R agonist SKF81297. The researchers then showed there was a causal relationship between internalization and cAMP accumulation by treating cultured striatal neurons with dynasore, a dynamin inhibitor to block endocytosis. Dynasore reduced the effect of dopamine mediated cAMP elevation indicating that D1R activation and endocytosis is responsible for cellular cAMP accumulation. This study showed that D1R endocytosis supports rapid dopaminergic neurotransmission through the early endocytic pathway.

\section{PLASTICITY}

The use of fluorescent biosensors in the field of neuroscience can lead to discoveries of previously undefined roles for signal transduction pathways. The synapse is the primary site of neurotransmission which undergoes a great deal of remodeling known as synaptic plasticity. The cAMP/PKA pathway regulates changes at the pre- and post-synapse through PKA phosphorylation of synaptic membrane components as well as cAMP responsive element binding protein (CREB) mediated gene transcription (Kandel, 2012). Modulation of normal synaptic processes leads to changes in cognition and behavior such as aggression, fear, anxiety, and learning and memory (Wallace et al., 2009; Liu et al., 2014). In 1991, a WD repeat actin binding protein called coronin 1 was identified in D. discoideum (de Hostos et al., 1991). Absence of coronin 1 in transgenic mice lead to defects in synaptic plasticity and behavior. Recently, 
Jayachandran et al. investigated the mechanism by which coronin 1 exerts its effects on the nervous system (Jayachandran et al., 2014). Investigation into the cellular localization of coronin 1 found that it localizes to excitatory synapses and not inhibitory synapses. Furthermore, PKA-dependent pre-synaptic LTP was absent in coronin 1 null mice indicating a defect in PKA signaling. Other experiments examining PKA phosphorylation of CREB and cAMP related physiological changes further suggested a change in cAMP/PKA signaling in coronin 1 null mice. To directly measure how cAMP is affected by coronin 1, the researchers used ICUE3 to monitor CAMP production in the coronin 1 deficient cell line Mel JuSo. Treatment with isoproterenol led to a minimal increase in cAMP whereas co-transfection of ICUE3 with a coronin 1 expression plasmid produced a robust increase in the cyan to yellow emission ratio. By combining the above data and data from protein-protein interaction experiments, the researchers concluded that coronin 1 potentiates cAMP/PKA signaling by positively interacting with the G-protein subunit Gas.

\section{ADVANCES IN THE FIELD BRAIN SLICE AND LIVE-ANIMAL IMAGING}

From shedding light on the kinetics of neuromodulatory signaling events to uncovering new aspects of signal transduction, fluorescent biosensors have been an invaluable tool for neuroscience research. Over the past decade, FRET-based biosensors have been used to dissect the intricacies of cyclic nucleotide signaling in the nervous system with unprecedented detail. Second messenger signaling is now being studied in more physiologically relevant samples like brain slices and transgenic animals with FRET-based biosensors (Calebiro et al., 2009; Thunemann et al., 2013). Polito and colleagues recently published a thorough protocol for imaging biosensors like AKAR in brain slices (Polito et al., 2014). These techniques are more demanding as they require intensive sample preparation and advanced equipment to preserve the spatial resolution afforded by single cell imaging experiments. The development of far-red and infrared biosensors will give researchers the ability to probe the biochemistry of cyclic nucleotides in deep tissues difficult to reach with current imaging methods (Shcherbakova and Verkhusha, 2013). Advances in microscopy tools are also an integral part of live brain imaging research. One such tool is fibered fluorescence microscopy that allows for a tissue imaging depth of up to $6 \mathrm{~mm}$ (Vincent et al., 2006). Using this technique, Vincent et al. were able to image peripheral nerve regeneration and calcium dynamics in central nervous nuclei of an anesthetized mouse. Although it has not yet been demonstrated, it is likely that the next wave of in vivo brain imaging will include studies of cyclic nucleotide signaling dynamics in live animals.

\section{PERTURBATION OF CYCLIC NUCLEOTIDE SIGNALING IN LIVING CELLS}

In addition to monitoring the biochemistry underlying neuronal activity, researchers are constantly developing innovative tools to perturb signaling molecules. One of these tools has been developed over the past decade in order to control the activity of neurons with light (Boyden et al., 2005). The technique is based on the light-driven ion channel called channelrhodopsin which can be genetically encoded, expressed and manipulated with light to depolarize or silence individual or groups of neurons. Optogenetics was a great leap forward in neuroscience allowing researchers to map circuits in brain slices and control the neural activity of transgenic animals. Similar tools have been developed to perturb cyclic nucleotide signaling. Light driven modulation of cyclic nucleotides began with the development of caged molecules that are activated through a photolysis reaction (Nargeot et al., 1983). Dimethoxy nitrobenzyl caged-cAMP was used in combination with AKAR in several different studies (Castro et al., 2013). Coumarinylmethyl caged cNMP derivatives developed over the past decade are more efficiently released under nondamaging light conditions (>360 nm wavelength). (Hagen et al., 2001; Geißler et al., 2003). New bis-carboxymethyl variants can allow for one and two-photon flash uncaging and could provide cyclic nucleotide release within deep tissue preparations (Hagen et al., 2005).

Neuronal cyclic nucleotide research has benefited from the implementation of genetically encodable photoactivatable adenylyl cyclase enzymes (PAC). This valuable addition to the toolbox allows for active user-controlled manipulation of cAMP and cGMP levels in specific locations within the cell. These bluelight (450 nm wavelength) sensitive nucleotidyl cyclases respond to light via conformational changes in the light-oxygen-voltage (LOV) or sensors of blue light using FAD (BLUF) photoreceptor protein domains. EuPAC from Euglena gracilis was the first to have a demonstrated PAC activity. It was used to show for example that focal stimulation of intracellular cAMP can steer the growth of Xenopus spinal commissural axons (Nicol et al., 2011). However, it suffered from high dark activity and poor heterologous cell expression due to its large size (Iseki et al., 2002). bPAC, also known as BlaC, was discovered in Beggiatoa sp. It was significantly smaller than EuPAC with low dark state activity which could be tuned by manipulating the level of expression (Ryu et al., 2010; Stierl et al., 2011). Stierl et al. used bPAC to successfully induce activity in cultured hippocampal neurons co-transfected with cyclic nucleotide gated ion channel. Ryu et al. further engineered it into a photoactivatable guanylyl cyclase, BlaG, by introducing three mutations into the region responsible for substrate binding. $\mathrm{BlaG}$ is the first photoactivatable guanylyl cyclase. More recently, mPAC was discovered in Microcoleus chthonoplastes (Raffelberg et al., 2013). This enzyme uses a LOV domain rather than the BLUF domain as in EuPAC and bPAC and has a greater AC activity in both the dark and light-activated state. The in vivo application of the aforementioned PACs is limited by the low tissue penetrance of the blue light needed to activate them. To increase tissue penetration, researchers developed a near-infrared adenylyl cyclase (IlaC) by fusing a bacteriophytochrome BphG1 to a bacterial adenylate cyclase CyaB1. IlaC was expressed in Caenorhabditis elegans cholinergic neurons and was shown to induce locomotion with exposure to red light $(650 \mathrm{~nm})$ as measured by the number of body bends per minute (Ryu et al., 2014). Given the spectral separation, IlaC opens up the possibility for PACs to be combined with biosensors for real-time manipulation and monitoring of cAMP and cGMP signaling pathways. 


\section{COMPUTATIONAL MODELING AND SIMULATION}

A fundamental question in cyclic nucleotide research is the maintenance of specific signaling in the midst of multiple inputs and outputs. For example, within neurons there is substantial cross-regulation of cAMP, cGMP and $\mathrm{Ca}^{2+}$. How do multiple signaling pathways interact to form a coherent output instead of chaos? Despite the advances in monitoring and manipulating cyclic nucleotides, we still do not have a clear understanding and additional tools such as mathematic modeling are needed to understand the intricacies of second messenger crosstalk and compartmentation (Saucerman et al., 2014). Significant efforts by different laboratories have been directed towards building mechanistic models to drive research hypotheses. One such study has started to reveal how a neuron's cell shape, topological distribution of biological components, and enzyme kinetics allows a cAMP/PKA microdomain to translate into a gradient of MAPK activity (Neves et al., 2008). The use of genetically encoded biosensors for imaging cyclic nucleotide signaling dynamics, either one at a time or better yet, in a co-imaging mode for multiple parameters, can help obtain more quantitative information, thereby facilitating model development and testing (Ni et al., 2011). Computational modeling efforts are aided by modeling software such as NeuroRD. These programs allow integration and simulation of structural data, enzyme kinetics, and diffusion rates for signaling networks. A guide to using NeuroRD is available with detailed information on developing and analyzing computational models of neuronal signaling networks (Blackwell et al., 2013). This tool has been used to demonstrate the role of PDE4D in maintaining subcellular cAMP microdomains with the requirements of PDE4D being anchored in the bulk cytosol and regulated by PKA phosphorylation (Oliveira et al., 2010). Similarly, stochastic reaction-diffusion models of dopamine signaling in the dendrites have demonstrated the importance of subcellular PKA colocalization with other components of the pathway to determine spatial signaling specificity (Oliveira et al., 2012).

With the multitude of neuromodulatory inputs affecting cyclic nucleotide levels, signaling information needs to be encoded specifically to produce specific cellular responses. Encoding can occur on spatial, temporal, and digital levels using molecular distribution, enzyme kinetics, and signal thresholding to elicit various physiological outputs from cAMP and cGMP signal transduction (Rich et al., 2014). Our understanding of cyclic nucleotide signaling within the context of neuromodulation continues to grow, facilitated by advances in biosensor and imaging technology. An interdisciplinary approach combining experimental and computational strategies may pave the way for future discoveries.

\section{ACKNOWLEDGMENTS}

This work is supported by funding from the NSF GRFP (1232825) to Kirill Gorshkov and NIH (R01DK073368) to Jin Zhang. We also thank Jessica Yang for critical reading of this manuscript, as well as the other members of the Zhang Lab and the Johns Hopkins School of Medicine Department of Pharmacology and Molecular Sciences for support.

\section{REFERENCES}

Adams, S. R., Harootunian, A. T., Buechler, Y. J., Taylor, S. S., and Tsien, R. Y. (1991). Fluorescence ratio imaging of cyclic AMP in single cells. Nature 349, 694-697. doi: $10.1038 / 349694 \mathrm{a} 0$

Allen, M. D., and Zhang, J. (2006). Subcellular dynamics of protein kinase A activity visualized by FRET-based reporters. Biochem. Biophys. Res. Commun. 348, 716721. doi: 10.1016/j.bbrc.2006.07.136

Antoni, F. A. (2000). Molecular diversity of cyclic AMP signalling. Front. Neuroendocrinol. 21, 103-132. doi: 10.1006/frne.1999.0193

Aye-Han, N.-N., Allen, M. D., Ni, Q., and Zhang, J. (2012). Parallel tracking of cAMP and PKA signaling dynamics in living cells with FRET-based fluorescent biosensors. Mol. Biosyst. 8, 1435-1440. doi: 10.1039/c2mb05514g

Biswas, K. H., Sopory, S., and Visweswariah, S. S. (2008). The GAF domain of the cGMP-binding, cGMP-specific phosphodiesterase (PDE5) is a sensor and a sink for cGMP. Biochemistry 47, 3534-3543. doi: 10.1021/bi702025w

Blackwell, K. T., Wallace, L. J., Kim, B., Oliveira, R. F., and Koh, W. (2013). Modeling spatial aspects of intracellular dopamine signaling. Methods Mol. Biol. 964, 6175. doi: 10.1007/978-1-62703-251-3_5

Bonnot, A., Guiot, E., Hepp, R., Cavellini, L., Tricoire, L., and Lambolez, B. (2014). Single-fluorophore biosensors based on conformation-sensitive GFP variants. FASEB J. 28, 1375-1385. doi: 10.1096/fj.13-240507

Boyden, E. S., Zhang, F., Bamberg, E., Nagel, G., and Deisseroth, K. (2005). Millisecond-timescale, genetically targeted optical control of neural activity. Nat. Neurosci. 8, 1263-1268. doi: 10.1038/nn1525

Calebiro, D., Nikolaev, V. O., Gagliani, M. C., de Filippis, T., Dees, C., Tacchetti, C., et al. (2009). Persistent cAMP-signals triggered by internalized Gprotein-coupled receptors. PLoS Biol. 7:e1000172. doi: 10.1371/journal.pbio.100 0172

Castro, L. R., Brito, M., Guiot, E., Polito, M., Korn, C. W., Hervé, D., et al. (2013). Striatal neurones have a specific ability to respond to phasic dopamine release. J. Physiol. 591, 3197-3214. doi: 10.1113/jphysiol.2013.252197

Castro, L. R., Gervasi, N., Guiot, E., Cavellini, L., Nikolaev, V. O., PaupardinTritsch, D., et al. (2010). Type 4 phosphodiesterase plays different integrating roles in different cellular domains in pyramidal cortical neurons. J. Neurosci. 30, 6143-6151. doi: 10.1523/JNEUROSCI.5851-09.2010

Cheng, P.-L., and Poo, M.-M. (2012). Early events in Axon/Dendrite polarization. Annu. Rev. Neurosci. 35, 181-201. doi: 10.1146/annurev-neuro-061010-113618

Conti, M., and Richter, W. (2014). "Phosphodiesterases and cyclic nucleotide signaling in the CNS," in Cyclic-Nucleotide Phosphodiesterases in the Central Nervous System, eds N. J. Brandon and A. R. West (John Wiley and Sons, Inc.), $1-46$.

Dao, K. K., Teigen, K., Kopperud, R., Hodneland, E., Schwede, F., Christensen, A. E., et al. (2006). Epacl and cAMP-dependent protein kinase holoenzyme have similar cAMP affinity, but their cAMP domains have distinct structural features and cyclic nucleotide recognition. J. Biol. Chem. 281, 21500-21511. doi: 10. 1074/jbc.m603116200

de Hostos, E. L., Bradtke, B., Lottspeich, F., Guggenheim, R., and Gerisch, G. (1991). Coronin, an actin binding protein of Dictyostelium discoideum localized to cell surface projections, has sequence similarities to G protein beta subunits. EMBO J. 10, 4097-4104.

del Puerto, A., Díaz-Hernández, J.-I., Tapia, M., Gomez-Villafuertes, R., Benitez, M. J., Zhang, J., et al. (2012). Adenylate cyclase 5 coordinates the action of ADP, P2Y1, P2Y13 and ATP-gated P2X7 receptors on axonal elongation. J. Cell Sci. 125, 176-188. doi: 10.1242/jcs.091736

Depry, C., Allen, M. D., and Zhang, J. (2011). Visualization of PKA activity in plasma membrane microdomains. Mol. Biosyst. 7, 52-58. doi: 10. 1039/c0mb00079e

DiPilato, L. M., Cheng, X., and Zhang, J. (2004). Fluorescent indicators of cAMP and Epac activation reveal differential dynamics of cAMP signaling within discrete subcellular compartments. Proc. Natl. Acad. Sci. U S A 101, 1651316518. doi: 10.1073/pnas.0405973101

DiPilato, L. M., and Zhang, J. (2009). The role of membrane microdomains in shaping [small beta]2-adrenergic receptor-mediated cAMP dynamics. Mol. Biosyst. 5, 832-837. doi: 10.1039/b823243a

Erard, M., Fredj, A., Pasquier, H., Beltolngar, D.-B., Bousmah, Y., Derrien, V., et al. (2013). Minimum set of mutations needed to optimize cyan fluorescent proteins for live cell imaging. Mol. Biosyst. 9, 258-267. doi: 10.1039/c2mb $25303 \mathrm{~h}$ 
Esseltine, J. L., and Scott, J. D. (2013). AKAP signaling complexes: pointing towards the next generation of therapeutic targets? Trends Pharmacol. Sci. 34, 648-655. doi: 10.1016/j.tips.2013.10.005

Geißler, D., Kresse, W., Wiesner, B., Bendig, J., Kettenmann, H., and Hagen, V. (2003). DMACM-caged adenosine nucleotides: ultrafast phototriggers for ATP, ADP and AMP activated by long-wavelength irradiation. Chembiochem 4, 162170. doi: $10.1002 /$ cbic. 200390027

Gervasi, N., Hepp, R., Tricoire, L., Zhang, J., Lambolez, B., Paupardin-Tritsch, D., et al. (2007). Dynamics of protein kinase A signaling at the membrane, in the cytosol and in the nucleus of neurons in mouse brain slices. J. Neurosci. 27, 2744-2750. doi: 10.1523/jneurosci.5352-06.2007

Giese, K. P., and Mizuno, K. (2013). The roles of protein kinases in learning and memory. Learn. Mem. 20, 540-552. doi: 10.1101/lm.028449.112

Hagen, V., Bendig, J., Frings, S., Eckardt, T., Helm, S., Reuter, D., et al. (2001). Highly efficient and Ultrafast Phototriggers for cAMP and cGMP by using long-wavelength UV/Vis-activation. Angew. Chem. Int. Ed Engl. 40, 1045-1048. doi: 10.1002/1521-3773(20010316)40:6<1045::aid-anie10450>3.0.co;2-f

Hagen, V., Dekowski, B., Nache, V., Schmidt, R., Geißler, D., Lorenz, D., et al. (2005). Coumarinylmethyl esters for ultrafast release of high concentrations of cyclic nucleotides upon one- and two-photon photolysis. Angew. Chem. Int. Ed Engl. 44, 7887-7891. doi: 10.1002/anie.200502411

Harootunian, A. T., Adams, S. R., Wen, W., Meinkoth, J. L., Taylor, S. S., and Tsien, R. Y. (1993). Movement of the free catalytic subunit of cAMP-dependent protein kinase into and out of the nucleus can be explained by diffusion. Mol. Biol. Cell 4, 993-1002. doi: 10.1091/mbc.4.10.993

Herbst, K. J., Coltharp, C., Amzel, L. M., and Zhang, J. (2011). Direct activation of Epac by sulfonylurea is isoform selective. Chem. Biol. 18, 243-251. doi: 10. 1016/j.chembiol.2010.12.007

Honda, A., Adams, S. R., Sawyer, C. L., Lev-Ram, V., Tsien, R. Y., and Dostmann, W. R. (2001). Spatiotemporal dynamics of guanosine 3/,5/-cyclic monophosphate revealed by a genetically encoded, fluorescent indicator. Proc. Natl. Acad. Sci. U S A 98, 2437-2442. doi: 10.1073/pnas.051631298

Iseki, M., Matsunaga, S., Murakami, A., Ohno, K., Shiga, K., Yoshida, K., et al. (2002). A blue-light-activated adenylyl cyclase mediates photoavoidance in Euglena gracilis. Nature 415, 1047-1051. doi: 10.1038/4151047a

Jayachandran, R., Liu, X., Bosedasgupta, S., Müller, P., Zhang, C. L., Moshous, D., et al. (2014). Coronin 1 regulates cognition and behavior through modulation of cAMP/protein kinase A signaling. PLoS Biol. 12:e1001820. doi: 10.1371/journal. pbio. 1001820

Kandel, E. R. (2012). The molecular biology of memory: cAMP, PKA, CRE, CREB1, CREB-2 and CPEB. Mol. Brain 5:14. doi: 10.1186/1756-6606-5-14

Klarenbeek, J. B., Goedhart, J., Hink, M. A., Gadella, T. W., and Jalink, K. (2011). A mTurquoise-based cAMP sensor for both FLIM and ratiometric read-out has improved dynamic range. PLoS One 6:e19170. doi: 10.1371/journal.pone. 0019170

Kotowski, S. J., Hopf, F. W., Seif, T., Bonci, A., and von Zastrow, M. (2011). Endocytosis promotes rapid dopaminergic signaling. Neuron 71, 278-290. doi: 10. 1016/j.neuron.2011.05.036

Lim, W. K. (2007). GPCR drug discovery: novel ligands for CNS receptors. Recent Pat. CNS Drug Discov. 2, 107-112. doi: 10.2174/157488907780832689

Lissandron, V., Terrin, A., Collini, M., D’alfonso, L., Chirico, G., Pantano, S., et al. (2005). Improvement of a FRET-based Indicator for cAMP by linker design and stabilization of donor-acceptor interaction. J. Mol. Biol. 354, 546-555. doi: 10. 1016/j.jmb.2005.09.089

Liu, X., Gu, Q. H., Duan, K., and Li, Z. (2014). NMDA receptor-dependent LTD is required for consolidation but not acquisition of fear memory. J. Neurosci. 34, 8741-8748. doi: 10.1523/JNEUROSCI.2752-13.2014

Liu, S., Li, Y., Kim, S., Fu, Q., Parikh, D., Sridhar, B., et al. (2012). Phosphodiesterases coordinate cAMP propagation induced by two stimulatory $\mathrm{G}$ proteincoupled receptors in hearts. Proc. Natl. Acad. Sci. U S A 109, 6578-6583. doi: 10. 1073/pnas.1117862109

Marley, A., Choy, R. W., and von Zastrow, M. (2013). GPR88 reveals a discrete function of primary cilia as selective insulators of GPCR cross-talk. PLoS One 8:e70857. doi: 10.1371/journal.pone.0070857

Mongillo, M., Mcsorley, T., Evellin, S., Sood, A., Lissandron, V., Terrin, A., et al. (2004). Fluorescence resonance energy transfer-based analysis of cAMP dynamics in live neonatal rat cardiac myocytes reveals distinct functions of compartmentalized phosphodiesterases. Circ. Res. 95, 67-75. doi: 10.1161/ 01.res.0000134629.84732.11
Nargeot, J., Nerbonne, J. M., Engels, J., and Lester, H. A. (1983). Time course of the increase in the myocardial slow inward current after a photochemically generated concentration jump of intracellular cAMP. Proc. Natl. Acad. Sci. U S A 80, 2395-2399. doi: 10.1073/pnas.80.8.2395

Nausch, L. W., Ledoux, J., Bonev, A. D., Nelson, M. T., and Dostmann, W. R. (2008). Differential patterning of cGMP in vascular smooth muscle cells revealed by single GFP-linked biosensors. Proc. Natl. Acad. Sci. U S A 105, 365-370. doi: 10. 1073/pnas.0710387105

Neves, S. R. (2012). Modeling of spatially-restricted intracellular signaling. Wiley Interdiscip. Rev. Syst. Biol. Med. 4, 103-115. doi: 10.1002/wsbm.155

Neves, S. R., Tsokas, P., Sarkar, A., Grace, E. A., Rangamani, P., Taubenfeld, S. M., et al. (2008). Cell shape and negative links in regulatory motifs together control spatial information flow in signaling networks. Cell 133, 666-680. doi: 10.1016/j. cell.2008.04.025

Newman, R. H., Fosbrink, M. D., and Zhang, J. (2011). Genetically encodable fluorescent biosensors for tracking signaling dynamics in living cells. Chem. Rev. 111, 3614-3666. doi: 10.1021/cr100002u

Ni, Q., Ganesan, A., Aye-Han, N. N., Gao, X., Allen, M. D., Levchenko, A., et al. (2011). Signaling diversity of PKA achieved via a Ca2+-cAMP-PKA oscillatory circuit. Nat. Chem. Biol. 7, 34-40. doi: 10.1038/nchembio.478

Nicol, X., Hong, K. P., and Spitzer, N. C. (2011). Spatial and temporal second messenger codes for growth cone turning. Proc. Natl. Acad. Sci. U S A 108, 13776-13781. doi: 10.1073/pnas.1100247108

Niino, Y., Hotta, K., and Oka, K. (2009). Simultaneous live cell imaging using dual FRET sensors with a single excitation light. PLoS One 4:e6036. doi: 10. 1371/journal.pone.0006036

Niino, Y., Hotta, K., and Oka, K. (2010). Blue fluorescent cGMP sensor for multiparameter fluorescence imaging. PLoS One 5:e9164. doi: 10.1371/journal. pone.0009164

Nikolaev, V. O., Bünemann, M., Hein, L., Hannawacker, A., and Lohse, M. J. (2004). Novel single chain cAMP sensors for receptor-induced signal propagation. J. Biol. Chem. 279, 37215-37218. doi: 10.1074/jbc.c400302200

Nikolaev, V. O., Bünemann, M., Schmitteckert, E., Lohse, M. J., and Engelhardt, S. (2006a). Cyclic AMP imaging in adult cardiac myocytes reveals farreaching $\beta 1$-adrenergic but locally confined $\beta 2$-adrenergic receptor-mediated signaling. Circ. Res. 99, 1084-1091. doi: 10.1161/01.res.0000250046.69 918.d5

Nikolaev, V. O., Gambaryan, S., and Lohse, M. J. (2006b). Fluorescent sensors for rapid monitoring of intracellular cGMP. Nat. Methods 3, 23-25. doi: 10. 1038/nmeth816

Norris, R. P., Ratzan, W. J., Freudzon, M., Mehlmann, L. M., Krall, J., Movsesian, M. A., et al. (2009). Cyclic GMP from the surrounding somatic cells regulates cyclic AMP and meiosis in the mouse oocyte. Development 136, 1869-1878. doi: $10.1242 /$ dev. 035238

Oliveira, R. F., Kim, M., and Blackwell, K. T. (2012). Subcellular location of PKA controls striatal plasticity: stochastic simulations in spiny dendrites. PLoS Comput. Biol. 8:e1002383. doi: 10.1371/journal.pcbi.1002383

Oliveira, R. F., Terrin, A., Di Benedetto, G., Cannon, R. C., Koh, W., Kim, M., et al. (2010). The role of type 4 phosphodiesterases in generating microdomains of cAMP: large scale stochastic simulations. PLoS One 5:e11725. doi: 10. 1371/journal.pone.0011725

Polito, M., Klarenbeek, J., Jalink, K., Paupardin-Tritsch, D., Vincent, P., and Castro, L. R. (2013). The NO/cGMP pathway inhibits transient cAMP signals through the activation of PDE2 in striatal neurons. Front. Cell. Neurosci. 7:211. doi: 10. 3389/fncel.2013.00211

Polito, M., Vincent, P., and Guiot, E. (2014). Biosensor imaging in brain slice preparations. Methods Mol. Biol. 1071, 175-194. doi: 10.1007/978-1-62703-622$1 \_14$

Ponsioen, B., Zhao, J., Riedl, J., Zwartkruis, F., van der Krogt, G., Zaccolo, M., et al. (2004). Detecting cAMP-induced Epac activation by fluorescence resonance energy transfer: Epac as a novel cAMP indicator. EMBO Rep. 5, 1176-1180. doi: 10.1038/sj.embor.7400290

Raffelberg, S., Wang, L., Gao, S., Losi, A., Gärtner, W., and Nagel, G. (2013). A LOV-domain-mediated blue-light-activated adenylate (adenylyl) cyclase from the cyanobacterium Microcoleus chthonoplastes PCC 7420. Biochem. J. 455, 359-365. doi: 10.1042/BJ20130637

Rich, T. C., Webb, K. J., and Leavesley, S. J. (2014). Can we decipher the information content contained within cyclic nucleotide signals? J. Gen. Physiol. 143, 17-27. doi: 10.1085/jgp.201311095 
Russwurm, M., Mullershausen, F., Friebe, A., Jäger, R., Russwurm, C., and Koesling, D. (2007). Design of fluorescence resonance energy transfer (FRET)-based cGMP indicators: a systematic approach. Biochem. J. 407, 69-77. doi: 10. 1042/bj20070348

Ryu, M. H., Kang, I. H., Nelson, M. D., Jensen, T. M., Lyuksyutova, A. I., SiltbergLiberles, J., et al. (2014). Engineering adenylate cyclases regulated by nearinfrared window light. Proc. Natl. Acad. Sci. U S A 111, 10167-10172. doi: 10. 1073/pnas.1324301111

Ryu, M. H., Moskvin, O. V., Siltberg-Liberles, J., and Gomelsky, M. (2010). Natural and engineered photoactivated nucleotidyl cyclases for optogenetic applications. J. Biol. Chem. 285, 41501-41508. doi: 10.1074/jbc.M110.177600

Saito, K., Chang, Y. F., Horikawa, K., Hatsugai, N., Higuchi, Y., Hashida, M., et al. (2012). Luminescent proteins for high-speed single-cell and whole-body imaging. Nat. Commun. 3:1262. doi: 10.1038/ncomms2248

Sample, V., DiPilato, L. M., Yang, J. H., Ni, Q., Saucerman, J. J., and Zhang, J. (2012). Regulation of nuclear PKA revealed by spatiotemporal manipulation of cyclic AMP. Nat. Chem. Biol. 8, 375-382. doi: 10.1038/nchembio.799

Sample, V., Newman, R. H., and Zhang, J. (2009). The structure and function of fluorescent proteins. Chem. Soc. Rev. 38, 2852-2864. doi: 10.1039/b913033k

Sato, M., Hida, N., Ozawa, T., and Umezawa, Y. (2000). Fluorescent indicators for cyclic GMP based on cyclic GMP-dependent protein kinase Ialpha and green fluorescent proteins. Anal. Chem. 72, 5918-5924. doi: 10.1021/ac0006167

Saucerman, J. J., Greenwald, E. C., and Polanowska-Grabowska, R. (2014). Mechanisms of cyclic AMP compartmentation revealed by computational models. J. Gen. Physiol. 143, 39-48. doi: 10.1085/jgp.201311044

Selvaratnam, R., Mazhab-Jafari, M. T., Das, R., and Melacini, G. (2012). The autoinhibitory role of the EPAC hinge helix as mapped by NMR. PLoS One 7:e48707. doi: 10.1371/journal.pone.0048707

Shcherbakova, D. M., and Verkhusha, V. V. (2013). Near-infrared fluorescent proteins for multicolor in vivo imaging. Nat. Methods 10, 751-754. doi: 10. 1038/nmeth.2521

Shelly, M., Cancedda, L., Lim, B. K., Popescu, A. T., Cheng, P.-L., Gao, H., et al. (2011). Semaphorin3A regulates neuronal polarization by suppressing axon formation and promoting dendrite growth. Neuron 71, 433-446. doi: 10.1016/j. neuron.2011.06.041

Shelly, M., Lim, B. K., Cancedda, L., Heilshorn, S. C., Gao, H., and Poo, M.-M. (2010). Local and long-range reciprocal regulation of cAMP and cGMP in Axon/Dendrite formation. Science 327, 547-552. doi: 10.1126/science.117 9735

Stierl, M., Stumpf, P., Udwari, D., Gueta, R., Hagedorn, R., Losi, A., et al. (2011). Light modulation of cellular cAMP by a small bacterial photoactivated adenylyl cyclase, bPAC, of the soil bacterium Beggiatoa. J. Biol. Chem. 286, 1181-1188. doi: 10.1074/jbc.M110.185496

Taly, A. (2013). Novel approaches to drug design for the treatment of schizophrenia. Expert Opin. Drug Discov. 8, 1285-1296. doi: 10.1517/17460441.2013.821108

Terrin, A., Di Benedetto, G., Pertegato, V., Cheung, Y.-F., Baillie, G., Lynch, M. J., et al. (2006). PGE1 stimulation of HEK293 cells generates multiple contiguous domains with different [cAMP]: role of compartmentalized phosphodiesterases. J. Cell Biol. 175, 441-451. doi: 10.1083/jcb.200605050
Thunemann, M., Wen, L., Hillenbrand, M., Vachaviolos, A., Feil, S., Ott, T., et al. (2013). Transgenic mice for cGMP imaging. Circ. Res. 113, 365-371. doi: 10. 1161/CIRCRESAHA.113.301063

Vincent, P., Maskos, U., Charvet, I., Bourgeais, L., Stoppini, L., Leresche, N., et al. (2006). Live imaging of neural structure and function by fibred fluorescence microscopy. EMBO Rep. 7, 1154-1161. doi: 10.1038/sj.embor.7400801

Violin, J. D., DiPilato, L. M., Yildirim, N., Elston, T. C., Zhang, J., and Lefkowitz, R. J. (2008). $\beta 2$-adrenergic receptor signaling and desensitization elucidated by quantitative modeling of real time cAMP dynamics. J. Biol. Chem. 283, 29492961. doi: 10.1074/jbc.m707009200

Wallace, D. L., Han, M. H., Graham, D. L., Green, T. A., Vialou, V., Iñiguez, S. D., et al. (2009). CREB regulation of nucleus accumbens excitability mediates social isolation-induced behavioral deficits. Nat. Neurosci. 12, 200-209. doi: 10. $1038 / \mathrm{nn} .2257$

Zaccolo, M., De Giorgi, F., Cho, C. Y., Feng, L., Knapp, T., Negulescu, P. A., et al. (2000). A genetically encoded, fluorescent indicator for cyclic AMP in living cells. Nat. Cell Biol. 2, 25-29. doi: 10.1038/71345

Zaccolo, M., and Pozzan, T. (2002). Discrete Microdomains with high concentration of cAMP in stimulated rat neonatal cardiac myocytes. Science 295, 17111715. doi: $10.1126 /$ science. 1069982

Zaccolo, M., and Stangherlin, A. (2014). "Compartmentalization and regulation of cyclic nucleotide signaling in the CNS," in Cyclic-Nucleotide Phosphodiesterases in the Central Nervous System, eds N. J. Brandon and A. R. West (John Wiley and Sons, Inc.), 59-76.

Zhang, J., Hupfeld, C. J., Taylor, S. S., Olefsky, J. M., and Tsien, R. Y. (2005). Insulin disrupts beta-adrenergic signalling to protein kinase A in adipocytes. Nature 437, 569-573. doi: 10.1038/nature04140

Zhang, C. L., Katoh, M., Shibasaki, T., Minami, K., Sunaga, Y., Takahashi, H., et al. (2009). The cAMP sensor Epac2 is a direct target of antidiabetic sulfonylurea drugs. Science 325, 607-610. doi: 10.1126/science.1172256

Zhang, J., Ma, Y., Taylor, S. S., and Tsien, R. Y. (2001). Genetically encoded reporters of protein kinase a activity reveal impact of substrate tethering. Proc. Natl. Acad. Sci. U S A 98, 14997-15002. doi: 10.1073/pnas.211566798

Conflict of Interest Statement: The authors declare that the research was conducted in the absence of any commercial or financial relationships that could be construed as a potential conflict of interest.

Received: 27 August 2014; accepted: 04 November 2014; published online: 04 December 2014.

Citation: Gorshkov K and Zhang J (2014) Visualization of cyclic nucleotide dynamics in neurons. Front. Cell. Neurosci. 8:395. doi: 10.3389/fncel.2014.00395

This article was submitted to the journal Frontiers in Cellular Neuroscience.

Copyright (๑) 2014 Gorshkov and Zhang. This is an open-access article distributed under the terms of the Creative Commons Attribution License (CC BY). The use, distribution and reproduction in other forums is permitted, provided the original author(s) or licensor are credited and that the original publication in this journal is cited, in accordance with accepted academic practice. No use, distribution or reproduction is permitted which does not comply with these terms. 